\title{
Contamination des moules (Mytilus galloprovincialis) des côtes de la région de Dakar par les Hydrocarbures Aromatiques Polycycliques (HAPs)
}

\author{
Momar NDIAYE $^{1 *}$, Abdoulaye DIOP ${ }^{1}$, Ana GAGO-Martínez ${ }^{2}$, José Antonio et \\ Rodríguez VAZQUEZ ${ }^{2}$ \\ ${ }^{1}$ Département de Chimie - Faculté des Sciences et Techniques- Université Cheikh Anta DIOP de Dakar - \\ Sénégal. BP 5005, Tel: +221 776407660, Fax: +221338246318 \\ ${ }^{2}$ Departamento de Química Analítica e Alimentaria - Facultade de Química - \\ Universidade de Vigo, Marcosende s/n 36200. Vigo - España. Tel/fax: 34986812322 \\ *Auteur correspondant ; E-mail: momndiaye@hotmail.com, momndiaye@ucad.edu.sn
}

\section{RESUME}

Dans ce travail, nous avons étudié la contamination par les Hydrocarbures Aromatiques Polycycliques des moules récoltées au niveau des côtes de la région de Dakar. Les sites ont été choisis en fonction des activités qui y sont développées. L'extraction des HAPs a été faite au moyen d'un extracteur Soxhlet avec un mélange hexane/acétone et la purification est réalisée avec l'oxyde d'aluminium. Nous avons analysé l'extrait par un chromatographe de gaz couplé à un spectrographe de masse (GC/MS). Les résultats montrent que les teneurs en hydrocarbures évoluent de façon croissante en fonction du temps. Au port de Dakar, les concentrations passent de 5308,67 en 2007 à 17973,37 $\mu \mathrm{g} / \mathrm{kg}$ de poids sec, en 2008. L'évolution des hydrocarbures suit la même tendance dans les différents sites. Ainsi, le Port concentre la plus forte teneur, ensuite suivent les sites de Mbao et de Hann. Au niveau du port, la contamination proviendrait des dépôts de stockages des hydrocarbures mais également des opérations de transbordement du pétrole. La zone de Mbao est polluée par la présence de nombreuses usines dont une raffinerie qui a ces canaux directement ouvert sur la plage. Le site de Hann abrite un canal qui draine les eaux usées domestiques mais aussi les huiles de rejet des stations services. Ainsi, les moules deviennent un bon indicateur de la qualité des eaux mais également un danger pour la population, grande consommatrice de cette espèce. Ces études qui constituent un état de lieu doivent être poursuivies afin de fournir des données scientifiques de base aux décideurs politiques pour la recherche de solutions durables à la pollution de notre environnement.

() 2012 International Formulae Group. All rights reserved.

Mots clés: Hydrocarbures Aromatiques Polycycliques, pollution, moule, Soxhlet, CG/MS, Dakar, Sénégal.

\section{INTRODUCTION}

Les hydrocarbures Aromatiques Polycycliques (HAPs) sont issus des processus de combustion incomplète de combustibles basés sur le carbone (charbon, huile, bois et gaz). Ils sont persistants, bioaccumulables et très stables dans l'environnement. Leur toxicité est surtout liée 
à un effet mutagénique. En effet ils peuvent se fixer sur les sites nucléophiles de l'ARN et de l'ADN, entrainant le développement de cancer (Xue et al., 2005; Zmirou et al., 2000).

Dans l'eau, ils sont hydrolysés et sont absorbés par les coquillages qui les accumulent (Filipkowska et al., 2005). L'espèce Mytilus galloprovincialis, est un mollusque bivalve à corps mou non segmenté. Elle est enveloppée par un manteau formé de deux grands lobes qui entourent le corps et qui ont sécrété la coquille calcaire bivalve (Raoux et al., 1993 ; Schönitzer et al., 2007). La moule est une espèce sessile, suspensivore et peut filtrer jusqu'à 100 à 200 litres d'eau par jour. Elle est capable d'opérer un tri concernant la nature et la taille des particules qui pénètrent dans la cavité palléale. Elle se nourrit de phytobenthos, de phytoplancton et de débris organiques. Elle absorbe beaucoup de substances durant son existence et peut donner des indications sur l'état de pollution $\mathrm{du}$ milieu. L'analyse de sa chair peut renseigner sur le degré de pollution de l'eau par les hydrocarbures aromatiques polycycliques et sur leur évolution dans le temps (Lamoureux et al., 1999). Ces produits de la mer, susceptibles de se retrouver dans les aliments, deviennent une source de contamination pour l'homme.

Il devient de ce fait important de contrôler les teneurs de certains hydrocarbures présents dans l'environnement pour prévenir une éventuelle contamination de la population.

Dans ce travail, nous avons étudié l'évolution en fonction du temps de la teneur des Hydrocarbures Aromatiques Polycycliques (HAPs) dans des échantillons de moules prélevés dans trois sites des côtes de la région de Dakar (Hann, M'Bao et les alentours du Port de Dakar).

\section{MATERIEL ET METHODES \\ Sites étudiés}

Les sites bordent l'océan atlantique et sont choisis pour les activités qui s'y déroulent. Les moules sont récoltées en 2007 et 2008 dans les stations de prélèvement de Mbao, Hann et du port de Dakar (Figure 1). La localité de Mbao (M1) abrite une Société Africaine de Raffinage du pétrole (SAR) et une centrale électrique. La plage de Hann (M2) est un lieu de débarquement des produits halieutiques. Elle abrite une usine de textile, des usines de transformation des produits de la mer et le canal Est qui draine les eaux usées domestiques. Le port de Dakar (M3) est actuellement en plein essor, les opérations de transbordement du pétrole sont fréquentes. De là, partent les pipelines qui alimentent les différentes sociétés d'hydrocarbures de la capitale.

\section{Produits utilisés}

Tous les réactifs utilisés sont de qualité «pour analyse» ou qualité nécessaire pour la chromatographie. L'hexane est de marque Panréac. C'est un solvant apolaire utilisé pour l'extraction des hydrocarbures. L'acétone est de marque Panréac. C'est un solvant polaire utilisé pour le lavage de la verrerie et pour l'extraction des hydrocarbures. L'oxyde d'aluminium $\mathrm{Al}_{2} \mathrm{O}_{3}$ (150 mesh, $58 \AA$, Aldrich) a été activé à $450{ }^{\circ} \mathrm{C}$ puis désactivé à $10 \%$ avec de l'eau millipore. Il est utilisé comme phase solide pour la chromatographie de partage. Le sulfate de sodium anhydre $\mathrm{Na}_{2} \mathrm{SO}_{4}$ (Panréac) est désactivé à $550{ }^{\circ} \mathrm{C}$ avant toute utilisation. Il sert à éliminer les traces d'eau. La solution standard contient les 16 Hydrocarbures Aromatiques Polycycliques (HAPs) (Tableau 1) dissous dans un mélange méthanol/chlorure de méthylène (1:1). On effectue une première dilution $(1 / 10)$ dans du méthanol, pour ensuite, par dilutions successives obtenir les autres solutions. Elles se conservent au réfrigérateur.

\section{Préparation des échantillons}

Les moules analysées dans cette étude, ont été récoltées à la main avant d'être stockées sous une température située en dessous de $0{ }^{\circ} \mathrm{C}$. 
Les moules sont détachées délicatement de leur support et sont épurées par immersion pendant 24 heures dans de l'eau de mer décantée, issue du site de prélèvement. Puis elles sont décoquillées et leur chair égouttée sur filtre Büchner en prenant toutes les précautions pour éviter la contamination de l'échantillon.

Ensuite, elles sont séchées à l'étuve à $40{ }^{\circ} \mathrm{C}$ pendant 3 à 4 jours avant d'être finement homogénéisées à l'aide d'un broyeur, pour obtenir des diamètres de particules de $60 \mu \mathrm{m}$ environ.

\section{Extraction des hydrocarbures}

L'extraction des HAPs a été faite au moyen d'un extracteur Soxhlet (Fernandez et al., 1996 ; Franco et al., 2006 ; Kanaly et al., 2000 ; Porte et al., 2000; Wilcke, 2000 ).

Nous avons mélangé $1 \mathrm{~g}$ d'échantillon de moule traité et $1 \mathrm{~g}$ de sulfate de sodium anhydre dans une cartouche en cellulose. L'extraction a été faite pendant 8 heures avec un mélange n-hexane/acétone $(1: 1, \mathrm{~V} / \mathrm{V})$. La température est réglée de manière à obtenir un reflux toutes les $15 \mathrm{~min}$.

\section{Purification des extraits}

L'extrait est passé à un courant d'azote pour éliminer le solvant. Le résidu est repris ensuite avec de l'hexane. Pour la purification, on élue l'extrait avec $40 \mathrm{~mL}$ d'hexane en utilisant comme phase stationnaire une colonne remplie d'oxyde d'aluminium désactivé à $10 \%$.

La phase de purification est réalisée par chromatographie d'adsorption, pour éliminer les substances organiques co-extraites, notamment les lipides. Il s'agit d'une méthode de séparation des constituants d'un mélange par migration dans un dispositif constitué de la phase stationnaire et de la phase mobile. Dans notre cas, l'alumine $\left(\mathrm{Al}_{2} \mathrm{O}_{3}\right)$ a servi de phase solide (Scott et al., 2004). La phase mobile est un mélange de deux solvants: l'un polaire (acétone), l'autre apolaire (hexane). On réalise alors un gradient d'élution.
Cependant, l'étape la plus importante est le remplissage de la colonne, pour une bonne séparation. Mais c'est également la plus délicate car le remplissage doit être le plus homogène possible et exempt de bulle d'air. Les surfaces inférieure et supérieure de l'adsorbant doivent être parfaitement horizontales.

L'éluant obtenu est concentré à $0,5 \mathrm{~mL}$ à l'aide d'un courant d'azote. Les HAPs sont enfin repris dans $1 \mathrm{~mL}$ d'hexane et gardés à l'obscurité à $4{ }^{\circ} \mathrm{C}$.

\section{Concentration}

L'extrait est concentré à l'aide d'un évaporateur rotatif, à $40{ }^{\circ} \mathrm{C}$ et sous pression réduite, jusqu'à obtenir un volume de $5 \mathrm{~mL}$ environ. Cet appareil permet d'éliminer rapidement un solvant volatil par évaporation. Le principe est basé sur l'abaissement du point d'ébullition avec la pression.

Les traces d'eaux résiduelles du concentrât peuvent être éliminées par addition de sulfate de sodium anhydre dans le ballon de l'évaporateur. L'extrait déshydraté est ensuite transféré dans un tube gradué, en opérant lentement de manière à ce que le sulfate de sodium reste au fond du ballon. Ce dernier est rincé avec 3 à $4 \mathrm{~mL}$ de solvant propre qui sont rajoutés dans le tube. A l'aide d'un courant d'azote, l'extrait organique est évaporé à sec. La solution est reprise avec 1 à $2 \mathrm{~mL}$ de solvant.

\section{Conditions opératoires}

L'extrait est analysé par un chromatographe de gaz couplé à un spectromètre de masse (GC/MS), qui offre la possibilité de doser les Hydrocarbures Aromatiques Polycycliques, notamment ceux qui ne sont pas fluorescents. Différents modes de détections sont mis en œuvre, dont le couplage à un spectromètre de masse (CG/MS).

Le Tableau 2 regroupe les conditions utilisées pour optimiser les performances de l'appareil. 


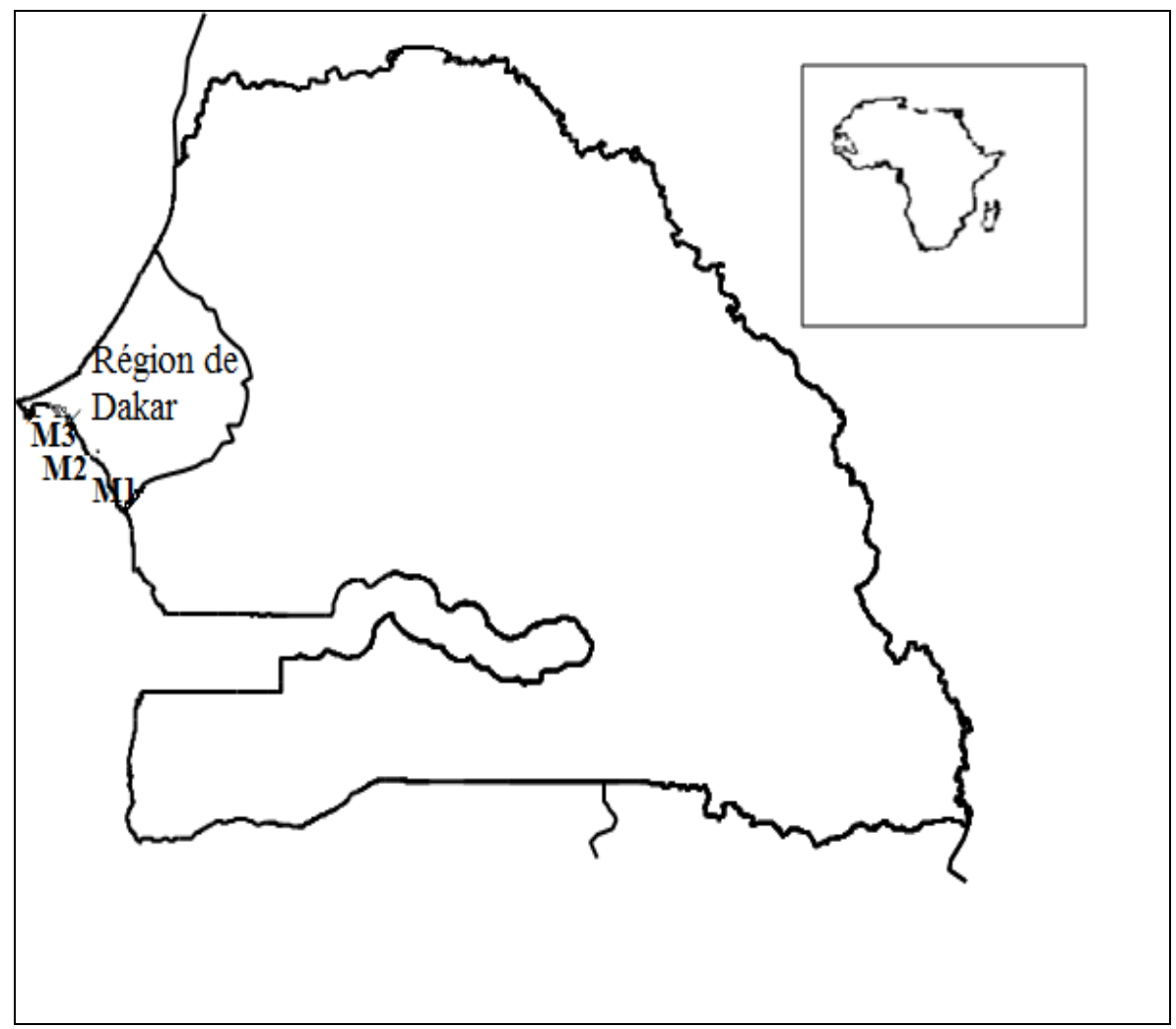

Figure 1: Carte des zones d'échantillonnage: M1(Mbao), M2 (Hann) et M3 (Port de Dakar).

\section{RESULTATS ET DISCUSSION}

La détection a été réalisée d'abord en mode Scan, qui permet d'avoir le temps de rétention et le spectre de masse de chaque composé. Ainsi, on dispose de "l'emprunte digitale" de tous les hydrocarbures. Puis, le mode SIM est utilisé pour diminuer les produits interférents et augmenter la limite de détection. La Figure 2 montre la séparation d'un mélange standard pur d'hydrocarbures. Tous les HAPs sont détectés. La Figure 3 représente un chromatogramme des hydrocarbures présents dans un échantillon de moule, après l'étape d'extraction.
Les moules, espèces sessiles, peuvent renseigner sur l'état de contamination du milieu.

Les résultats obtenus, dans les trois sites (Hann, Mbao et Port) pour les différentes campagnes, par la méthode de la courbe de calibration, sont rassemblés dans les Tableaux 3 et 4. En 2007: Les hydrocarbures de faibles poids moléculaires sont détectés dans les moules récoltées au niveau des trois sites. A l'exception du chrysène, ceux de poids moléculaires élevés ne sont pas retenus par les bivalves. Cependant, le naphtalène est plus concentré dans la zone de Hann alors que le chrysène $y$ est absent. 
En 2008, les hydrocarbures de faibles poids moléculaires sont détectés dans les différents sites. Cependant, le fluorène et l'anthracène ne sont pas dosés à Hann. Ce dernier composé n'est pas non plus trouvé à Mbao. Ceux de poids moléculaires élevés (BbF, BkF, BaP, Ipy, DahA et BghiP) sont absents des zones de Hann et de Mbao, alors qu'ils sont très bien représentés au niveau du Port et de Soumbédioune. La Figure 4 montre l'évolution des HAPs, pendant les années 2007 et 2008, en fonction des sites.

L'analyse des histogrammes montre que l'année 2008 présente les teneurs les plus élevées, dans les différents sites. Ainsi, la pollution par les hydrocarbures évolue de façon croissante dans les moules, en fonction $\mathrm{du}$ temps. Le Port concentre les valeurs maximales $(5308,67$ et $17973,37 \mu \mathrm{g} / \mathrm{kg}$ poids sec en 2007 et 2008 respectivement) suivi de Mbao et de Hann. Les valeurs maximales, enregistrées au niveau du Port, sont probablement le fait des opérations de transbordement du pétrole. En effet, c'est de là que partent les pipelines qui alimentent les différentes sociétés d'hydrocarbure de la capitale. Mais également le Port, en plein essor, reçoit beaucoup de navires qui $y$ effectuent la maintenance de leur moteur. En effet, la majeure partie de la pollution aux hydrocarbures proviendrait des nombreux dégazages et déballastages illégaux (2,3 millions de tonnes de pétrole par an), mais aussi aux rejets industriels (Ndiaye et al., 2006). Le dégazage est l'opération qui consiste à débarrasser des cuves de carburant ou des cuves de pétrole brut, les gaz qui y subsistent une fois la cuve vidée. Dans un navire, la solution la plus pratique et la moins coûteuse consiste à dilater les gaz à la vapeur, à ventiler et à rincer la cuve avec de l'eau. Les rejets opérationnels de tous les navires sont ainsi comparables au rejet dans la nature d'huile de vidange d'une voiture ou d'un camion. La seule différence est qu'il ne s'agit pas de centaines de millions de personnes susceptibles de jeter quelques litres d'huile à chaque fois mais de dizaines de milliers de navires déversant plusieurs tonnes d'hydrocarbures à chaque opération. Le déballastage désigne le déchargement des eaux de lestage du navire, des résidus de cargaison liquide et des résidus de fonctionnement. Cependant, ces concentrations sont en deçà des teneurs trouvées dans les ports de Vendres, en France, et de Barcelone, en Espagne, situés sur la Méditerranée (Baumard et al., 1999).

Dans la région de Dakar, les déchets d'hydrocarbures proviennent également des vidanges des voitures. Ces déchets sont recyclés, en partie, par la société de régénération des hydrocarbures $(\mathrm{SRH})$ dont la capacité de traitement est limitée. Le reste des hydrocarbures est soit enfoui, soit déversé à la mer par le biais des canaux qui débouchent à l'Est de la région de Dakar à la plage de Hann et à l'Ouest à celle de Soumbédioune. En plus, ces deux sites connaissent un afflux des pirogues à moteur, qui y débarquent des produits halieutiques (Ndiaye et al., 2006). De même, la baie de Hann compte un total de 99 entreprises, susceptibles de participer à sa pollution.

$\mathrm{Au}$ niveau du site de Mbao, la contamination pourrait être causée par les unités industrielles installées dans la zone franche, et surtout la société de raffinage du pétrole (SAR) qui a ses canaux ouverts directement sur la plage. Ainsi, les moules constituent un bon indicateur de la pollution marine, mais aussi un danger pour la chaîne alimentaire. En effet ces espèces, très consommées actuellement à Dakar, accumulent les hydrocarbures Aromatiques Polycycliques. 


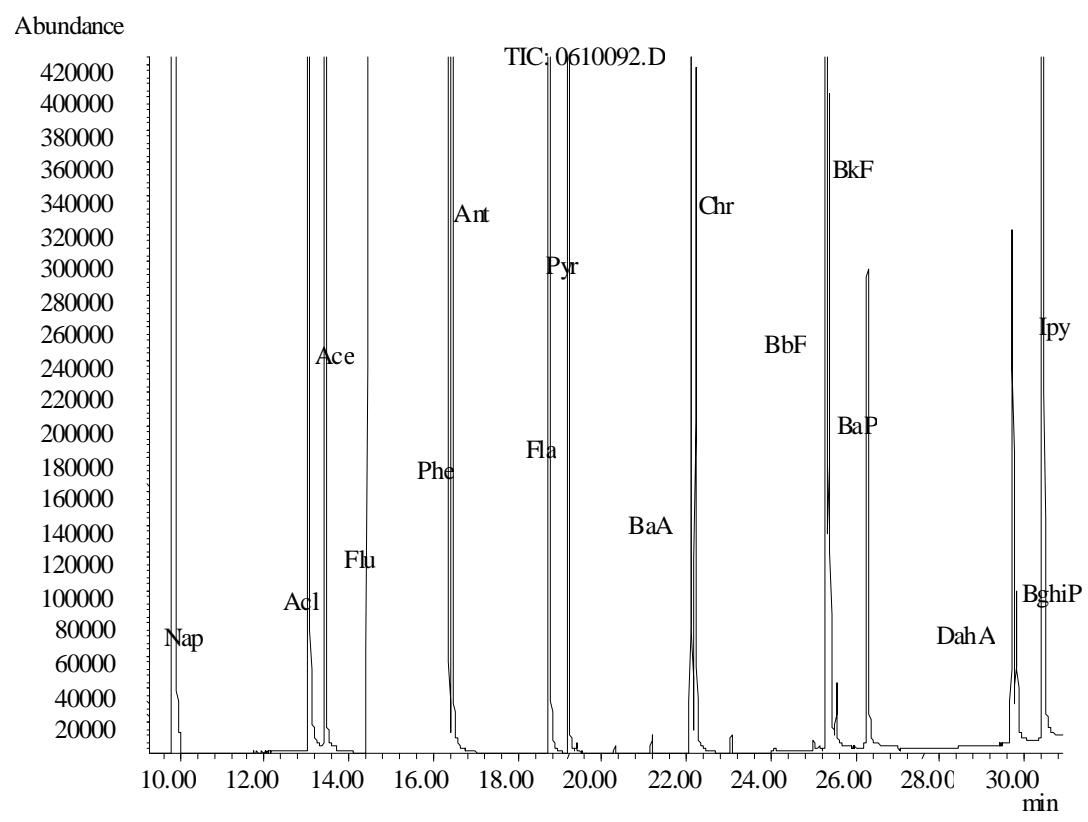

Figure 2: Chromatogramme d'une solution standard des 16 HAPs en mode SIM.

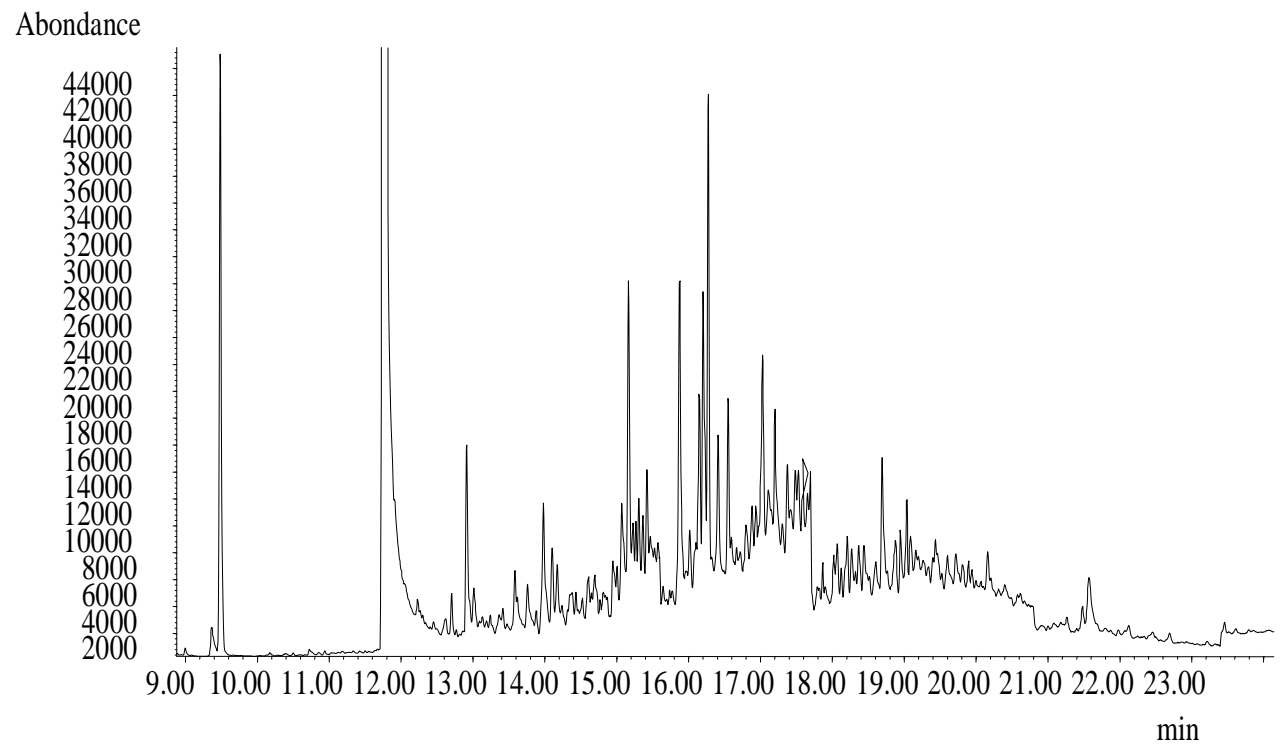

Figure 3: Chromatogramme d'un échantillon de moule. 


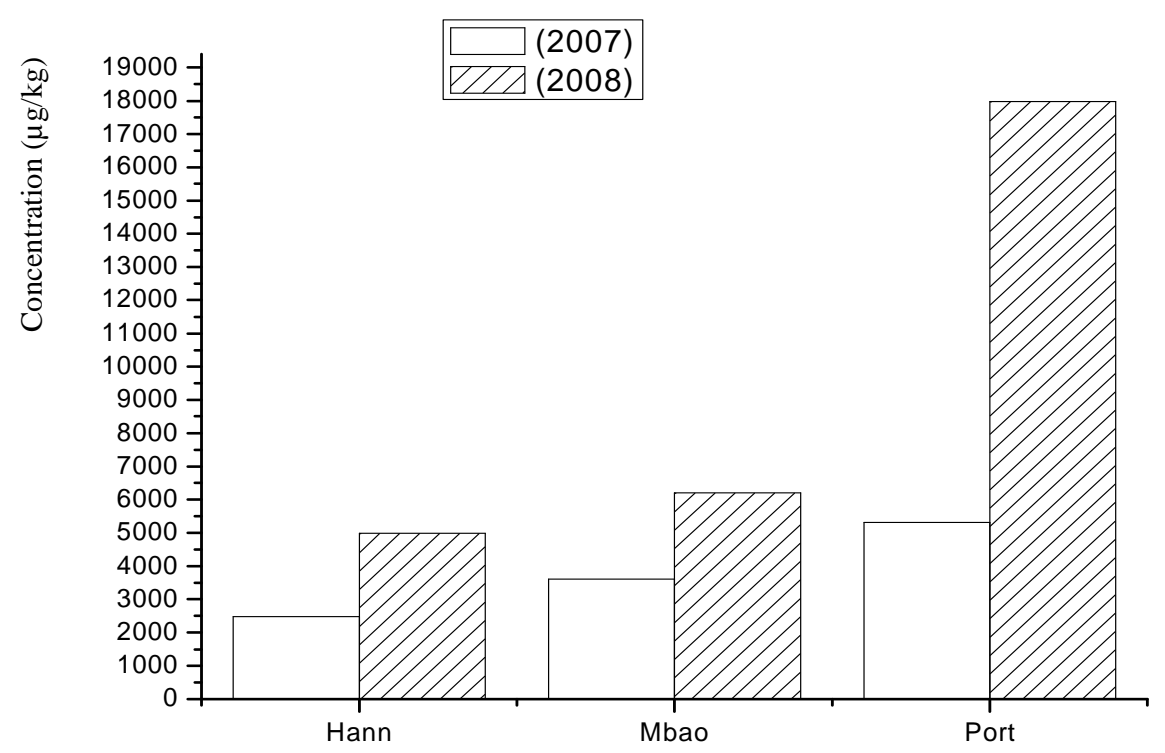

Figure 4: Evolution de la teneur des HAPs pour chaque site, en 2007 et 2008.

Tableau 1: Solution standard des 16 HAPs.

\begin{tabular}{lcc}
\hline Composés & $\begin{array}{c}\text { Concentration } \\
(\boldsymbol{\mu} \mathbf{g} / \mathbf{m L})\end{array}$ & Déviation standard \\
\hline Naphtalène (Nap) & 1021 & 1,3 \\
Acénaphtylène (Acl) & 2043 & 2 \\
Acénaphtène (Ace) & 1022 & 0,6 \\
Fluorène (Flu) & 203,6 & 0,29 \\
Phénanthrène (Phe) & 104,9 & 0,07 \\
Anthracène (Ant) & 101,5 & 0,25 \\
Fluoranthène (Fla) & 207,7 & 3,43 \\
Pyrène (Pyr) & 104,2 & 1,84 \\
Benzo[a]anthracène (BaA) & 102,4 & 0,07 \\
Chrysène (Chr) & 101,9 & 0,11 \\
Benzo[b]fluoranthène (BbF) & 204,9 & 0,22 \\
Benzo[k]fluoranthène (BkF) & 101,5 & 0,13 \\
Benzo[a]pyrène (BaP) & 102,1 & 0,19 \\
Indeno[1,2,3-c,d)pyrène (Ipy) & 102,2 & 0,18 \\
Dibenzo[a,h]anthracène (DahA) & 204,1 & 0,01 \\
Benzo[g,h,i]pérylène (BghiP) & 204,4 & 0,3 \\
\hline
\end{tabular}


Tableau 2: Paramètres du système CG/MS optimisés pour la mesure des HAPs.

\begin{tabular}{|l|l|}
\hline Système CG /MS & HP 6890 Series CG $-5973 \mathrm{MS}$ \\
\hline Colonne Capillaire & $30 \mathrm{~m}$ x $0,25 \mathrm{~mm} \times 0,25 \mu \mathrm{m}$ d.i. $\mathrm{HP}-5 \mathrm{MS}$ \\
\hline Programme de la température du four & \\
\hline & \\
\hline Injection & $60{ }^{\circ} \mathrm{C}$ \\
\hline Ionisation & Splitless: $250{ }^{\circ} \mathrm{C}$ \\
\hline Température de l'interface & Impact électronique: $70 \mathrm{eV}$ \\
\hline Température du détecteur de masse & $280{ }^{\circ} \mathrm{C}$ \\
\hline Mode de détection & Source d'ionisation: $250{ }^{\circ} \mathrm{C}$ \\
\hline Ions sélectionnés $\mathrm{m} / \mathrm{z}$ & Température quadripolaire: $100{ }^{\circ} \mathrm{C}$ \\
\hline Vitesse du flux de la phase mobile & Scan \\
\hline Temps de Splitless & $100-300$ \\
\hline
\end{tabular}

Tableau 3: Teneur des HAPs dans les moules recueillies en 2007.

\begin{tabular}{lccc}
\hline \multirow{2}{*}{ Composés } & \multicolumn{3}{c}{ Concentration $(\boldsymbol{\mu g} / \mathbf{k g}$ poids sec $)$} \\
\cline { 2 - 4 } & Hann & Mbao & Port \\
\hline Nap & 406,54 & 77,34 & 112,49 \\
Acl & 726,37 & 1559,34 & 1761,07 \\
Ace & 801,63 & 918,07 & 1311,10 \\
Flu & ND & ND & 332,11 \\
Phe & 296,87 & 204,22 & 586,55 \\
Ant & ND & ND & 158,83 \\
Fla & 161,54 & 542,29 & 437,51 \\
Pyr & 81,69 & 71,26 & 336,99 \\
BaA & ND & ND & ND \\
Chr & ND & 234,79 & 272,03 \\
BbF & ND & ND & ND \\
BkF & ND & ND & ND \\
BaP & ND & ND & ND \\
Ipy & ND & ND & ND \\
DahA & ND & ND & ND \\
BghiP & ND & ND & ND \\
Total & 2474,64 & $\mathbf{3 6 0 7 , 2 9}$ & $\mathbf{5 3 0 8 , 6 7}$ \\
\hline
\end{tabular}


Tableau 4: Teneur des HAPs dans les moules recueillies en 2008.

\begin{tabular}{lccc}
\hline Composés & \multicolumn{3}{c}{ Concentration $(\boldsymbol{\mu g} / \mathbf{k g}$ poids sec) } \\
\cline { 2 - 4 } & Hann & Mbao & Port \\
\hline Nap & 813,49 & 751,14 & 1194,78 \\
Acl & 1724,70 & 1731,34 & 2534,64 \\
Ace & 1010,02 & 1305,55 & 1512,53 \\
Flu & ND & 298,75 & 535,72 \\
Phe & 387,41 & 340,63 & 673,15 \\
Ant & ND & ND & 338,56 \\
Fla & 201,32 & 1140,06 & 1151,95 \\
Pyr & 200,84 & 118,12 & 618,25 \\
BaA & 329,52 & 265,41 & 625,84 \\
Chr & 314,97 & 250,80 & 578,51 \\
BbF & ND & ND & 512,37 \\
BkF & ND & ND & 679,55 \\
BaP & ND & ND & 1106,62 \\
Ipy & ND & ND & 1492,02 \\
DahA & ND & ND & 3322,80 \\
BghiP & ND & ND & 1096,08 \\
Total & $\mathbf{4 9 8 2 , 2 7}$ & $\mathbf{6 2 0 1 , 7 9}$ & $\mathbf{1 7 9 7 3 , 3 7}$ \\
\hline
\end{tabular}

\section{Conclusion}

Dans ce travail, nous avons étudié la distribution des Hydrocarbures Aromatiques Polycycliques dans des échantillons de moules récoltées dans les côtes de la région de Dakar. Les méthodes chromatographiques de haute résolution utilisées sont efficaces pour la séparation des HAPs, avec différents modes de détection. La chromatographie de gaz couplée à un spectromètre de masse permet d'analyser les composés à l'état de traces surtout ceux qui ne sont pas fluorescents. Les résultats obtenus montrent que les moules sont contaminées par les hydrocarbures, ce qui peut entrainer un problème de santé publique. De plus, le Port concentre les teneurs maximales du fait des activités qui y sont développées. Mais, également les moules récoltées au niveau du site de Hann, l'un des plus importants quais de débarquement des produits halieutiques, peuvent constituées un danger pour la population consommatrice. Ces études doivent être poursuivies afin de fournir des données scientifiques de base aux décideurs politiques pour la recherche de solutions durables à la maîtrise de la pollution de l'environnement. Les chercheurs, notamment les chimistes, ont donc un rôle prépondérant à jouer dans cette recherche de solutions.

\section{REFERENCES}

Baumard P, Budzinski H, Garrigues P, Dizer H, Hansen PD. 1999. Polycyclic aromatic hydrocarbons in recent sediments and mussels (Mytilus edulis) from the Western Baltic Sea: occurrence, bioavailability and seasonal variations. Mar. Environ. Res., 47: 17-47.

Fernandez I, Dachs J, Bayona JM. 1996. Application of experimental design approach to the optimization of 
supercritical fluid extraction of polychlorinated biphenyls and polycyclic aromatic hydrocarbons. Journal of Chromatography A, 719: 77-85.

Filipkowska A, Lubecki L, Kowalewska G. 2005. Polycyclic aromatic hydrocarbon analysis in different matrices of the marine environment. Analytica Chimica Acta, 547: 243-254.

Franco MA, Viñas L, Soriano JA, de Armas D, González JJ, Beiras R, Salas N, Bayona JM, Albaigés J. 2006. Spatial distribution and ecotoxicity of petroleum hydrocarbons in sediments from the Galician continental shelf (NW Spain) after the Prestige oil spill. Marine Pollution Bulletin, 53: 260-271.

Kanaly RA, Harayama S. 2000. Biodegradation of High-MolecularWeight Polycyclic Aromatic Hydrocarbons by Bacteria. Journal of Bacteriology, 182(8): 2059-2067.

Lamoureux E, Brownawell BJ. 1999. Chemical and biological availability of sediment-sorbed hydrophobic organic contaminants. Environ. Toxicol. Chem. 18(8): 1733-1741.

Ndiaye M, Diop A, Diouf B, Ndiaye A, Ndour C, Santos LM, Gago-Martinez A, Vazquez JAR. 2006. Etude du profil de contamination des sédiments marins à Dakar par les hydrocarbures aromatiques polycycliques. J. Soc. Ouest-Afr. Chim. 022: 105-113.

Porte C, Biosca X, Pastor D. 2000. The Aegean Sea oil Spill 2, temporal study of the hydrocarbons accumulation in bivalves. Environmental Science and Technology, 34(24): 5067-5075.

Raoux CY, Garrigues P. 1993. Mechanism model of polycyclic aromatic hydrocarbons contamination of marine coastal sediments from the Mediterranean sea. In Proceedings of the 13th International Symposium on Polynuclear Aromatic Hydrocarbons, Garrigues P, Lamotte M (eds). Gordon and Breach Publishers, 443 - 450.

Schönitzer V, Weiss IM. 2007. The structure of mollusc larval shells formed in the presence of the chitin synthase inhibitor Nikkomycin Z". BMC Structural Biology, 7: 7-71.

Scott AS, Allen DU, Stephen D, EmbsoMattingly. 2004. Environ. Sci. Technol., 38: 2987-2994.

Wilcke W. 2000. Polycyclic Aromatic Hydrocarbons (PAHs) in Soil - A Review. Journal Plant Nutr. Soil Science, 163: 229-248.

Zmirou D, Masclet P, Boudet C, Dor F, Déchenaux J. 2000. Personal exposure to atmospheric polycyclic aromatic hydrocarbons in a general adult population and lung cancer risk assessment. J. Occup. Environ. Med., 42(2): 121-126.

Xue W, Warshawsky D. 2005. Metabolic activation of polycyclic and heterocyclic aromatic hydrocarbons and DNA damage: a review. Toxicol. Appl. Pharmacol., 206(1): 73-93. 